\title{
Le « Parcours du Praticien» une application de l'apprentissage par problèmes à la formation médicale continue
}

\author{
Raymond Colin *, Pierre Reyes* *, Jean-Louis Bernard ** , Gérard Dongradi** *
}

\begin{abstract}
Résumé Contexte: "Le Parcours du Praticien » est un outil pédagogique original qui applique les principes de l'apprentissage par problèmes à la formation médicale continue (FMC). Méthode : Cet outil comporte trois documents : 1- Un scénario clinique divisé en plusieurs étapes; 2- Une série de questions correspondant à des objectifs pédagogiques repérables dans le scénario clinique ; 3- Un document de référentiels réunissant les données normatives actuelles reliées aux objectifs pédagogiques. La production de ces documents obéit à un processus impliquant de nombreux médecins généralistes et spécialistes, visant à identifier à propos du thème du Parcours les objectifs pertinents et à rédiger un scénario clinique crédible propre à l'identification des indices reliés aux objectifs. Le "Parcours du Praticien " est utilisé au cours d'un enseignement interactif en petits groupes, selon des modalités qui mettent en application les principes fondamentaux de la méthode des apprentissages par problèmes. Résultats : En France, 9 programmes regroupant 40 Parcours ont été réalisés et diffusés. Plus de $95 \%$ des médecins participants aux séances de FMC utilisant les Parcours ont apprécié favorablement la méthode. Conclusion : Le "Parcours du Praticien " est un nouvel outil de FMC particulièrement adapté au développement de l'expertise professionnelle.
\end{abstract}

Mots clés Formation médicale continue ; outil pédagogique ; apprentissage par problème.

Summary Background: "Le Parcours du Praticien " is an original pedagogic tool which applies the principles of problem - based learning for Continuing Medical Education (CME). Method: This tool contains three sections: 1-A clinical situation divided into various stages; 2 - A series of questions which correspond to the pedagogic objectives found in a clinical scenario ; 3- A section of references grouping current standard data related to educational objectives. The creation of these documents corresponds to a process which involves various general practitioners and specialists, with the aim of identifying the pertinent objectives as well as a believable clinical setting which are related to corresponding objective indices. The "Parcours du Praticien" is used during small group interactive courses according to the modalities that put into practice the fundamental principles of the problem-based learning. Results: In France, 40 "Parcours du Praticien " were regrouped into 9 programmes carried out and put into practice. More than $95 \%$ of the practitionners participating in the course using the "Parcours du Praticien " appreciated the method. Conclusion: "The Parcours du Praticien" is a new useful tool for CME well convenient in particular to develop competence in medical practice.

Key Words Continuing medical education ; pedagogic tool ; problem based learning.

Pédagogie Médicale 2001 ; 2 : 93-99.

\footnotetext{
* Département d'Hépato-Gastroentérologie et de Nutrition - Hôpital Charles Nicolle Rouen 76031 Cedex, France ** Institut de Formation et de Recherches sur I'Apprentissage de la Médecine (IFRAM), 13000 Marseille, France ** *ACALIS - Editions Médicales - 34 rue Camille Pelletan - 92309 Levallois-Perret, France Correspondance : Raymond Colin - Hôpital Charles Nicolle - Département d'Hépato-Gastroentérologie et de Nutrition CHU - 1 rue Germont - 76031 ROU日N Cedex - Tél. : + 33232888172 - Télécopie : + 33232888396 Courrier électronique : raymond.colin@chu-rouen.fr
} 


\section{Concepts et Innovations}

\section{Introduction}

L'utilisation de problèmes cliniques pour favoriser l'apprentissage des médecins s'est imposée comme un moyen de mettre en application les données actuelles issues de la recherche en psychologie cognitive ${ }^{1,2}$. Le terme " apprentissage par problèmes " (APP) regroupe diverses méthodes dont le champ actuel d'application est principalement la formation initiale des médecins ${ }^{3}$. Dans cette utilisation, le choix de la méthode doit être adapté au niveau des étudiants et aux objectifs visés. A titre d'exemple, l'APP centré sur l'analyse s'applique aux étudiants en début de cursus, et facilite l'acquisition et l'intégration des sciences fondamentales et cliniques ${ }^{4}$. Au contraire, l'apprentissage au raisonnement clinique est adapté au niveau des étudiants de deuxième cycle et cherche à développer une aptitude à utiliser des connaissances dans une démarche de pratique du raisonnement hypothético-déductif ${ }^{4}$.

Compte tenu de son intérêt pédagogique au cours de la formation initiale, il a été imaginé que le principe de l'APP pourrait s'appliquer à la formation médicale continue (FMC). Cette hypothèse a conduit à concevoir un outil pédagogique, "le Parcours du Praticien ", spécifiquement destiné à la FMC, inspiré de la méthode de l'APP, et élaboré selon un processus cherchant à garantir sa pertinences .

Dans cet article, nous décrirons successivement les principes généraux qui ont guidé la conception du "Parcours du Praticien », les documents pédagogiques, les modalités de production de ces documents, la méthodologie d'une séance de FMC utilisant cet outil, enfin l'état de développement actuel de cette méthode en France.

\section{Principes généraux}

Le « Parcours du Praticien " place les médecins participant à une séance d'enseignement en FMC devant une situation professionnelle sous la forme d'un scénario décrivant l'histoire clinique d'un malade. Celle-ci les conduit, à propos de ce cas clinique, à développer en petit groupe une réflexion sur leur pratique et à discuter les divers problèmes émanant de l'analyse du cas. Ce cas clinique comporte des indices qui sont reliés à des objectifs spécifiques préalablement déterminés.

Les participants peuvent ensuite élaborer une démarche diagnostique et proposer une prise en charge thérapeutique, sociale et psychologique du malade.
Un spécialiste de contenu agit comme personne ressource pour commenter la production collective du groupe, répondre à d'éventuelles questions au fur et à mesure du déroulement des étapes évolutives du cas clinique.

Les participants disposent à la fin de la séance d'un document dans lequel sont consignées les données scientifiques actuelles et les recommandations éventuelles de bonne pratique portant sur les objectifs pédagogiques suscités par le scénario clinique.

Ainsi conçue, la séance d'enseignement utilisant le " Parcours du Praticien » s'appuie sur les règles de base de la méthode de l'APP. En effet, l'apprentissage se fait à partir d'un support narratif d'une situation professionnelle; il est guidé par des objectifs spécifiques. Durant la séance d'enseignement, les participants sont actifs et autonomes; l'enseignant a un rôle d'animateur référent et très accessoirement de dispensateur de données. Les échanges des participants favorisent une réactivation des connaissances sur le thème abordé et suscitent un besoin de clarification et d'acquisition de nouveaux savoirs.

\section{Description de l'outil pédagogique}

Le « Parcours du Praticien » aborde un problème clinique de pratique courante, comme, par exemple, l'augmentation modérée chronique des transaminases, les troubles de la miction ou l'asthme de l'enfant.

Il comporte trois documents sous forme de groupes de feuilles reliées :

- Le Parcours proprement dit est un texte décrivant l'histoire clinique d'un malade, qui permet au participant de se placer dans la situation de témoins, leur permettant soit de s'identifier au praticien dont l'action est décrite, soit de la critiquer. Ce texte est séparé en trois ou quatre étapes successives, comportant au total trente à quarante points clés sous forme d'indices signalés par un graphisme particulier (caractère gras ou rouge) et par une numérotation. Ces points clés peuvent comporter à dessein des erreurs ou des imperfections telles que l'on peut les observer en pratique et que le Comité de rédaction a choisi de mettre en scène en raison de leur intérêt pédagogique.

- Les Questions du Praticien correspondent aux points clés numérotés. Il s'agit de susciter sur un mode interrogatif la curiosité des participants. Ces points clés sont en relation avec des objectifs pédagogiques 


\section{La douleur chez l'enfant - Atelier $n^{\circ} 1$ \\ 1. Douleur fracturaire chez un enfant de 6 ans}

\section{Le Parcours}

Arnaud, 6 ans, qui vient de faire une violente chute de vélo dans le parc voisin, est conduit au cabinet médical par sa mère; encore casqué il se tient le coude droit et pleure;

il n'y a pas eu perte de connaissance.

Une appréciation rapide de la douleur (1) est réalisée. Après un discours apaisant et un déshabillage délicat :

- l'examen du membre supérieur droit est très évocateur d'une fracture supracondylienne de l'humérus; il n'y a pas de signe de lésion vasculaire ou nerveuse;

- l'interrogatoire et l'examen physique systématiques (2) sont rassurants.

Pour traiter cette douleur fracturaire au cabinet,

- il est pratiqué une immobilisation à visée antalgique (3), - il est administré un médicament antalgique (4). On explique à l'enfant et ses parents la nature des lésions et la nécessité d'une prise en charge en milieu orthopédique. La fracture sera réduite sous anesthésie générale (5). Arnaud ne gardera pas un trop mauvais souvenir de son accident.

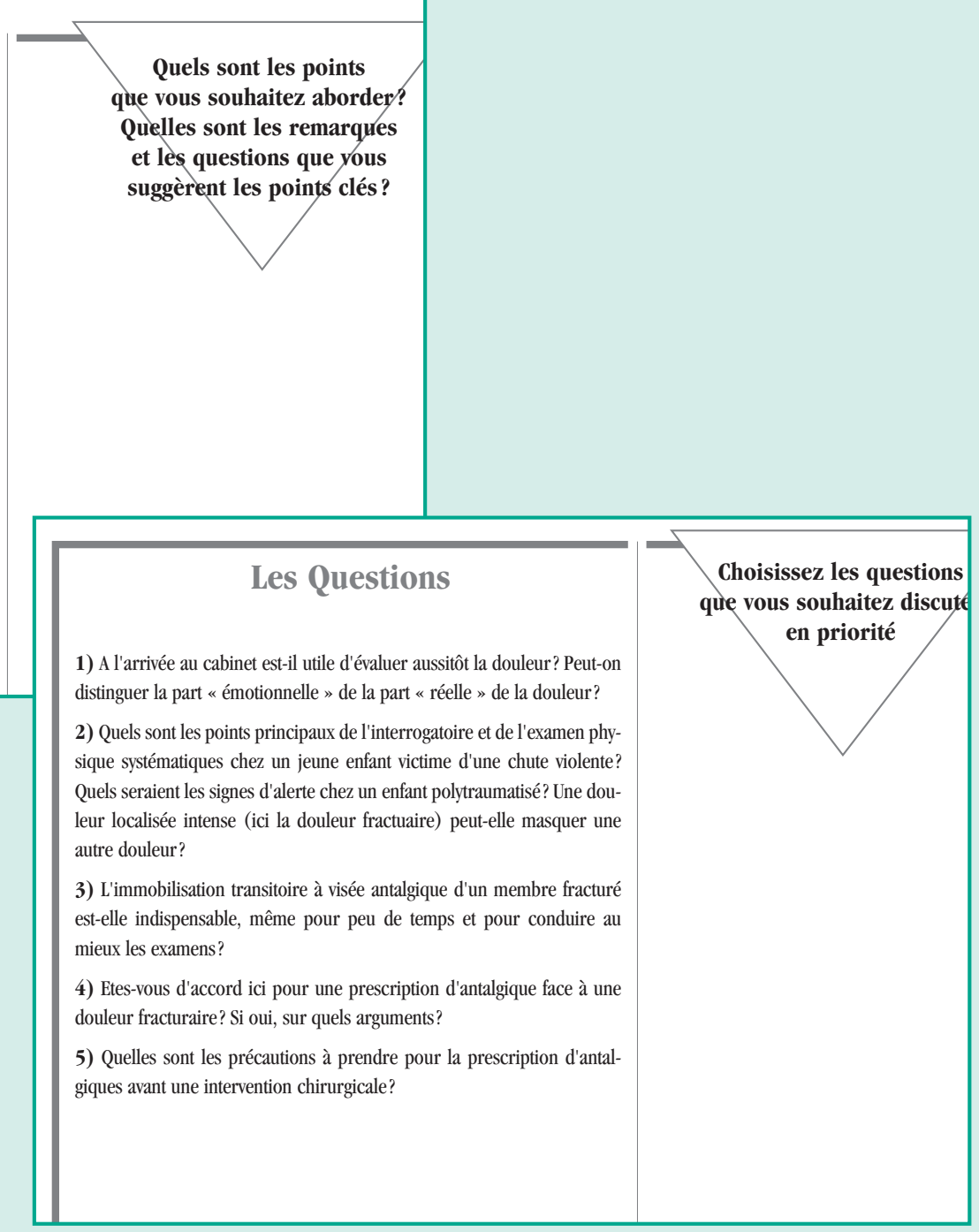

1) A l'arrivée au cabinet est-il utile d'évaluer aussitôt la douleur? Peut-on distinguer la part « émotionnelle " de la part « réelle » de la douleur?

Evaluation d'emblée

Dans une situation d'urgence, le médecin est naturellement préoccupé par l'établissement d'un diagnostic et la recherche de signes de gravité. Il doit aussi penser à, systématiquement, considérer le paramètre douleur dans sa réflexion clinique, sans nécessairement l'étayer d'emblée par une évaluation instrumentale précise, souvent inopportune et peu fiable dans un contexte fortement émotionnel. Un enfant sur deux fréquentant les urgences pédiatriques pour fracture ou entorse ressent une douleur forte (intensité équivalente à plus de 60 sur une EVA). On sait que la douleur est d'autant plus forte qu'il s'agit d'une fracture et non d'une entorse, que la fracture est proximale, qu'elle concerne le membre supérieur ${ }^{1}$

Comment apprécier?

Uneprésomption de douleurpeutsuffire dans un premiertemps, en se basant sur la nature des lésions constatées, sur les réactions provoquées par l'examen clinique. Dans la mesure du possible, éventuellement de façon légèrement différée après les premiers soins, une évaluation objective de l'intensité de la douleur est fort souhaitable.

L'âge de 5 ans représente un âge charnière pour l'évaluation de la douleur. En fonction du degré de compréhension et de verbalisation de l'enfant, une échelle d'auto-évaluation pourra ou non être utilisée

Partémotionnelle

Une réaction émotionnelle est habituelle dans les suites de tout traumatisme, tant chez l'enfant que chez son entourage. Chez l'enfant, ladouleur accidentelle brutale est sourced'uneanxiété qui renforce elle-même la perception douloureuse. Pour lui, la gravité de l'accident est liée à l'intensité de sa douleur, et la soulager équivaut à le guérir. Chez les parents, l'inquiétude sur le diagnostic et la gravité de la blessure, la souffrance del'enfant présente et future, un éventuel sentiment de culpabilité, sont autant defacteursémotionnels modulés parla culture et lapersonnalité de chacun, mais aussi par le contexte de l'accueil et de la prise en charge (attente, empathie)... Tout concourt ainsi à générer chezl'enfant un stress etune anxiété dontles symptômes sont très proches de ceux dela douleur aiguë (pleurs, cris, agitation, hostilité). Du fait de cette ambiguïté sémiologique, il 


\section{Concepts et Innovations}

\section{Tableau 1 \\ Déroulement d'un atelier de formation utilisant les « Parcours du Praticien»}

\section{Accueil :}

- remise des documents ;

- émargement dans un but d'accréditation;

- répartition des participants par groupes de 8 à 12 praticiens.

T 0 Introduction : présentation de la méthode pédagogique et des supports

T 5 minutes Evaluation : pré-test

T 15 minutes Début de la première étape du Parcours.
A) Sélection (6 minutes) :
- lecture du Parcours et annotations individuelles (3 minutes)
- lecture des questions et sélection individuelle (3 minutes)
B) Discussion (9 minutes) :
- échange entre les participants sur leurs pratiques à propos des points clés
- sélection des questions prioritaires
C) Discussion avec l'expert référent (20 minutes) :
- les points clés choisis en priorité par le groupe ;
- les autres points clés et objectifs majeurs de l'étape.

T 45 minutes Début de la deuxième étape du Parcours*.

T 70 minutes Début de la troisième étape du Parcours*.

T 100 minutes Fin de la discussion de la troisième étape du Parcours.

T 110 minutes Evaluation : post-test.

T 120 minutes Fin de l'atelier.

* les différentes étapes suivent un schéma identique à la première étape 
considérés comme pertinents par le comité de rédaction. Ils sont proposés et peuvent être retenus ou non pour une discussion plus approfondie entre participants et si nécessaire avec l'expert référent.

- Le document des référentiels réunit les réponses aux questions et les commentaires des points clés. Ce document intègre, chaque fois que possible, les données normatives actuelles telles que les conclusions de conférence de consensus, les recommandations nationales et internationales de bonne pratique, les données de la "Médecine basée sur les preuves ». Il peut comporter des tableaux récapitulatifs ou des arbres décisionnels. Il aborde aussi les points délicats de l'exercice médical, ceux pour lesquels il n'existe pas de données clairement déterminantes et de bonne pratique établie, ou pour lesquels coexistent plusieurs attitudes que le praticien pourra adopter sur la base de critères non objectifs. Les données importantes sont regroupées dans des encadrés. Ce document de référence peut être utilisé par l'expert. Il est remis à chaque participant à la fin de la séance ce qui lui permet s'il le conserve de s'y référer ultérieurement à propos de tel ou tel de ses malades.

\section{Modalités d'élaboration des documents}

La réalisation des supports pédagogiques est originale. Elle est conduite selon une procédure participative et interactive dont l'objectif principal est la recherche de la pertinence en vue de son utilisation comme outil de FMC.

Le choix des thèmes émane d'un consensus entre les promoteurs (Société Savante, Association de Formation, Industrie Pharmaceutique) et un panel de praticiens généralistes et spécialistes universitaires et non universitaires.

Les membres du Comité de rédaction, composé de médecins généralistes et spécialistes, assistent dans un premier temps à une série de réunions regroupant des médecins praticiens qui discutent, à propos du thème choisi, des difficultés rencontrées dans leur pratique. Ces divers groupes élaborent une première série d'objectifs pédagogiques qui servent de guides pour la rédaction d'un scénario initial. Ce scénario et les objectifs pédagogiques sont ensuite testés selon la méthode classique des " Parcours du Praticien " par de nouveaux groupes de médecins répartis sur l'ensemble du territoire, en présence d'un tuteur ayant une bonne connaissance de la méthode pédagogique, et par ailleurs professionnel dans la discipline, sans être nécessairement expert de contenu.

Ces réunions, au nombre de vingt à cinquante, appelées Séminaires de Rédaction, ont pour objectifs d'enrichir et de valider les points clés et d'évaluer la qualité de la rédaction du scénario. Chaque tuteur expert fournit au Comité de Rédaction, à l'issue de la séance à laquelle il a assisté, des critiques et suggestions pour rendre plus pertinent le scénario final et pour arrêter la liste définitive des objectifs pédagogiques.

Dans un dernier temps, les textes de références sont rédigés et validés par le Comité Scientifique avec l'aide des experts de contenu du programme. Un document d'évaluation destiné au pré-test et post-test, sous forme de vingt questions ouvertes courtes, est réalisé.

Au total cette procédure permet de faire émerger des objectifs pédagogiques pertinents pour chaque thème du fait de la diversité des statuts, des pratiques et de l'effectif des praticiens impliqués (analyse originale des besoins). Sa complexité nécessite organisation, rigueur et savoir-faire. Le processus d'élaboration offre en luimême à chaque participant une extraordinaire occasion de formation personnelle. Enfin, il aboutit à l'édition de documents adaptés aux attentes des médecins praticiens et conformes aux données scientifiques et aux pratiques professionnelles actuelles.

\section{Déroulement d'un atelier de formation utilisant le "Parcours du Praticien"}

Un atelier de FMC utilisant le "Parcours du Praticien " s'adresse à un groupe de huit à dix médecins ou à plusieurs groupes réunis dans une même salle.

Chaque table désigne un animateur chargé du bon déroulement de la séance. Un expert, généralement spécialiste universitaire ou non universitaire compétent dans le domaine du thème choisi, assiste à l'ensemble de l'atelier et intervient à la demande. Le déroulement d'une séance de formation est reproduit dans le tableau $n^{\circ} 1$. Une séance dure au total deux heures. 


\section{Concepts et Innovations}

\section{État de l'implantation actuelle des "Parcours du Praticien"}

Entre 1996 et 2000, neuf programmes nationaux sur des thématiques diverses ont été réalisés ou engagés, regroupant au total une quarantaine de Parcours. Ces Parcours ont été conçus à l'initiative ou sous la tutelle de Sociétés Savantes (Association Française d'Urologie, Société Française de Pédiatrie) ou d'Associations de Praticiens (Association Confédérale de Formation Médicale etc....). La réalisation pratique des programmes a été assurée par la société d'édition spécialisée, Acalis, propriétaire de la méthode et de l'outil Le Parcours du Praticien ${ }^{\oplus}$. Au plan du financement ces programmes ont été parrainés par des firmes pharmaceutiques qui ont largement contribué à la diffusion des programmes.

Dans le contexte actuel de la FMC en France, il n'a pas été possible à ce jour de réaliser de tels programmes pour le compte d'associations subventionnées par des institutions ou des organismes de FMC. L'indépendance scientifique a cependant constamment été respectée sous le contrôle des Comités de Rédaction et des Comités Scientifiques.

Il est difficile d'apprécier exactement le nombre de médecins ayant bénéficié d'un ou de plusieurs ateliers de FMC utilisant le Parcours du Praticien. Toutefois, l'Association Française d'Urologie, qui a élaboré un programme de onze Parcours couvrant l'ensemble de la discipline, a recensé plus de 8000 médecins ayant participé à au moins un atelier d'urologie.

Les fiches mesurant les indices d'évaluation de la méthode remplies à l'issue de chaque séance font apparaître de manière homogène un taux de satisfaction (satisfait ou très satisfait) de $95 \%$. Les participants qualifient la méthode de "pratique ", «active " et " attractive ".

Il pourrait être intéressant de tenter de transposer cet outil pédagogique et la méthode d'utilisation qui s'y rattache dans le milieu médical international moyennant une éventuelle adaptation notamment pour ce qui concerne les référentiels.

\section{Conclusion}

Le "Parcours du Praticien " est un outil pédagogique original destiné à la FMC. Il est adapté à une formation interactive en petits groupes. Son originalité réside d'une part, dans un processus de conception des documents qui en garantit la pertinence, d'autre part dans un format qui permet de respecter les principes fondamentaux d'une pédagogie adaptée à l'entretien et au développement de l'expertise professionnelle*.

* Les auteurs tiennent une documentation détaillée à la disposition des praticiens qui soubaiteraient appliquer cette méthode à leur action de formation continue.

\section{Références}

1. NormanGR, Schmidt HG. The psychological basis of problem-based learning : a review of the evidence. Academic Medicine 1992 ; 67 : 557565.

2. Schmidt H. Fondations of problem-based learning : some exploration notes. Medical Education $1993 ; 27: 422-432$.

3. Barrows HS. Problem-based learning applied to medical education. Springfield, Illinois USA : Southern Illinois University School of Medicine Publication, 1994.

4. Dumais B, Des Marchais JE. L'apprentissage par problèmes : le véhicule de la réforme pédagogique. In: Des Marchais JE. et Collaborateurs (Eds). Apprendre à devenir Médecin. Université de Sherbrooke : Canada. 1996 pp 83-117.

5. Honnorat C, Levasseur G. Quels besoins enseigner, quel besoin d'enseigner. Pédagogie Médicale $2001 ; 2: 26-30$. 


\section{Programmes de FMC réalisés de 1996 à 2000 selon les concepts et les méthodes du Parcours du Praticien}

1 Les Parcours du Praticien en Pathologie Digestive

Coordonnateur : Pr R. Colin (Rouen)

12 ateliers édités par Sanofi-Synthelabo

2 Les Parcours du Praticien en Urologie

Coordonnateurs : Pr F. Richard (Paris-La Pitié Salpétrière),

$\mathrm{Pr}$ Ph. Grise (Rouen), Dr J-P. Allègre (Valence)

11 ateliers édités par Sanofi-Synthelabo

sous l'égide de l'Association Française d'Urologie

3 La Douleur rachidienne chez l'Adulte

Coordonnateur : Pr J-M. Le Parc (Paris - Ambroise Paré)

2 ateliers édités par Théraplix

4 La Fièvre de l'Enfant

Coordonnateur : Pr J. Gaudelus (Paris - Bondy)

2 ateliers édités par Théraplix

5 Les Ateliers de l'Asthme

Coordonnateur : Pr A-B. Tonnel (Lille)

5 ateliers édités par Glaxo-Wellcome

sous l'égide de l'Association Confédérale pour la FMC

6 Athérosclérose : des facteurs de risque aux complications

Coordonnateur : Pr L. Guize (Paris - Broussais)

5 ateliers édités par Rhône-Poulenc Rorer

sous l'égide de l'Association Confédérale pour la FMC et de

l'Union nationale de Formation continue et d'évaluation en médecine cardiovasculaire

7 La Douleur chez l'Enfant

Coordonnateurs : Pr J-L. Bernard (Marseille), Dr P. Reyes (Marseille), Dr J. Stagnara (Lyon)

2 ateliers édités par Théraplix

sous l'égide de la Société Française de Pédiatrie

8 Pneumologie de l'Enfant

Direction : Pr J.-L. Bernard (Marseille), Dr P. Reyes (Marseille), Dr J. Stagnara (Lyon)

Coordonnateurs : Pr G. Dutau (Toulouse), Pr A. Grimfeld

(Paris Trousseau), Dr M. Pradal (Marseille)

2 ateliers édités sous par Théraplix

sous l'égide de la Société Française de Pédiatrie

9 Les Ateliers de Dermatologie

Coordonnateurs : Dr Ph. Beaulieu (Paris), Dr Véronique Gassia (Toulouse),

Pr Ph. Humbert (Besançon), Pr G. Lorette (Tours)

Ateliers en cours d'édition par les Laboratoires Leo

sous l'égide de la Fédération Française de Formation Continue en Dermato-Vénéréologie

et du Collège des Enseignants en Dermatologie de France 